\title{
Competitividad y emprendimiento: herramientas de crecimiento económico de un país
}

\section{Competitiveness and entrepreneurship: economic growth tools of a country}

Sandy Jahaira Gonzaga Añazco,

Tania Patricia Alaña Castillo, Andreina Inés González Ordóñez

Universidad Metropolitana del Ecuador, Ecuador

Autor para correspondencia: sandyjgonzagaa; talana@umet.edu.ec; andreinagonzalez09@gmail.com Fecha de recepción: 05 de Julio de 2017 - Fecha de aceptación: 12 de Agosto de 2017

Resumen: En este artículo, se presentan algunas teorías y supuestos fundamentales para el desarrollo de una cultura emprendedora, como justificación para potenciar procesos creativos e innovadores, capaces de generar productividad y desarrollo a partir de la creación de nuevos entes económicos a nivel local, regional y nacional. Pretende aclarar el constructo teórico del emprendimiento y la competitividad, identificar sus características fundamentales y resaltar la importancia de los mismos, como herramientas de desarrollo económico de los países. El emprendimiento ha sido estudiado desde la mirada de varias ciencias que se relacionan con el mismo, por ejemplo la economía, la psicología, la sociología y la antropología. Al principio, la economía estudió el emprendimiento buscando su relación con el desarrollo desde un punto de vista puramente económico. Posteriormente, la psicología, la sociología y la antropología desarrollaron estudios del emprendimiento desde una óptica social. Para desarrollar este objetivo se consideró el estudio y análisis de 16 referencias bibliográficas relacionadas con el emprendimiento y la competitividad, como elementos fundamentales de crecimiento económico sostenible de un país. El enfoque abordado es netamente conceptual y pretende clarificar conceptos e incentivar la investigación futura en relación con los emprendimientos y la competitividad, buscando determinar su importancia, principalmente en las economías emergentes.

Palabras Clave: competitividad; emprendimiento; emprendedor; desarrollo económico

Abstract: This article presents some theories and fundamental assumptions for the development of an entrepreneurial culture as a justification for promoting creative and innovative processes capable of generating productivity and development through the creation of new economic entities at the local, regional and national levels. It seeks to clarify the theoretical construct of entrepreneurship and competitiveness, to identify its fundamental characteristics and to emphasize the importance of them, as economic development tools of the countries. Entrepreneurship has been studied from the perspective of several related sciences, such as economics, psychology, sociology and anthropology. At first, the economy studied entrepreneurship seeking their relationship with development from a purely economic point of view. Subsequently, psychology, sociology and anthropology developed studies of entrepreneurship from a social perspective. To develop this objective, the study and analysis of 16 bibliographical references related to entrepreneurship and competitiveness were considered as fundamental elements of a country's sustainable economic growth. The approach is clearly conceptual and seeks to clarify concepts and encourage future research in relation to entrepreneurship and competitiveness, seeking to determine their importance, especially in emerging economies.

Key words: competitiveness; entrepreneurship; entrepreneurship; economic development 


\section{Introducción}

Los avances científicos y tecnológicos, la globalización del conocimiento y la economía, desafían a las instituciones tanto educativas como gubernamentales a promover procesos de investigación, innovación, creatividad y emprendimiento empresarial, en la solución de problemas de su entorno a partir de la aplicación del conocimiento.

Desde hace ya varias décadas se ha relacionado al emprendimiento con el descubrimiento de oportunidades de rentabilidad. Por ejemplo Cantillón en 1755 en sus publicaciones indicaba que el emprendimiento surge cuando se detectan posibles oportunidades que beneficiarían de manera positiva al negocio naciente (Cantillón, 1755).

Casson declara que las oportunidades de emprendimiento son aquellas situaciones en las que nuevos bienes, servicios, materias primas y métodos de organización, pueden ser vendidos e introducidos a un precio mayor que sus costos de producción (Casson, 1982)

Por otro lado Shane y Venkataraman, perfeccionan las definiciones antes mencionadas, indicando que el emprendedor es entonces quien descubre, evalúa y explota oportunidades rentables, tomando en cuenta el riesgo, alerta a las oportunidades y necesidad por la innovación (Shane \& Venkataraman, 2000).

Con respecto a lo anteriormente mencionado Madrid M. relaciona al emprendimiento con la competitividad e indica que esta se encuentra vinculada con la capacidad de innovar, la disponibilidad de mercados eficientes, la dotación y utilización eficiente de factores productivos y las características de la organización empresarial (Madrid, 2010).

Una empresa cuenta con mayor probabilidad supervivencia si mantiene incorporada en su estrategia corporativa al emprendimiento como herramienta de creación de riqueza. Al respecto se plantean que las nuevas empresas que entran en el mercado son generalmente pequeñas, y a pesar que éstas tienen menos probabilidad de sobrevivir, un crecimiento rápido provocado por la presencia de estrategias innovadoras, puede condicionar su supervivencia (Taymaz \& Köksal, 2006).

\section{Factores que afectan a la actividad emprendedora}

El emprendimiento es un mecanismo que mediante la innovación incrementa la productividad, con la disponibilidad de los recursos humanos y tecnológicos adecuados. Para lo cual es necesario analizar los factores que afectan a la actividad emprendedora como factor de crecimiento y competitividad: el ecosistema de los emprendimientos y los factores internos que en él intervienen.

El ecosistema de emprendimiento hace referencia al conjunto de actores tales como entidades gubernamentales, organizaciones públicas y privadas, entidades financieras, instituciones educativas, que interactúan formal o informalmente para conectar, mediar y gobernar el desempeño en un ambiente local de emprendimiento, facilitando la creación y el desarrollo sostenible de nuevas empresas (Mason \& Brown, 2014). 
La existencia de un ecosistema fortalecido es un factor que incide en la competitividad de un país, en este sentido, la generación de cultura emprendedora requiere de la presencia de una política pública en materia de emprendimiento que constituya la base para la formación de un ecosistema de emprendimiento (Soto Rodriguez, 2014).

Los factores internos tienen relación con las características propias de la empresa y pueden ser determinantes para su posicionamiento y sostenibilidad en el mercado, como tamaño, crecimiento, nivel de endeudamiento, entre otros.

\section{El papel del emprendedor}

Los emprendedores son individuos que innovan, identifican y crean oportunidades de negocios, administran de manera eficiente los recursos disponibles, con la finalidad de obtener los mejores beneficios de sus innovaciones en un medio incierto" (BÓVEDA, 2004).

Un emprendedor se caracteriza por hacer de la innovación una herramienta de desarrollo personal, ya que es capaz de hacer algo novedoso, de dar otro uso a algo que ya existe y así participar en la transformación de su propia vida y la de su entorno. Además, el emprendedor es aquel que tiene la capacidad para generar ideas, transformarlas, adaptarlas, proponer diversas alternativas y visualizar en un problema una oportunidad, por medio de la identificación de oportunidades de negocio.

Existen varias características que pueden destacar a un emprendedor, las mismas que aportan de manera significativa al desarrollo del espíritu empresarial en los individuos que las poseen, como por ejemplo el reconocimiento oportuno de sus errores, la optimización de recursos limitados como el tiempo, la constante búsqueda de soluciones creativas, alto espíritu colaborador, sin temor al cambio, necesidad de logro, disciplina, alta autoestima, trabajo por resultados, construcción de ambientes positivos frente a la iniciativa de un proyecto; además varios investigadores identifican el espíritu emprendedor con: dinamismo, creatividad, innovación, flexibilidad, crecimiento y riesgo.

Los emprendedores son aquellos que ven en las crisis posibles oportunidades de negocio y las saben aprovechar de manera eficiente, para obtener resultados económicos favorables. Los pilares fundamentales para emprender un negocio son: el liderazgo, el trabajo en equipo, la responsabilidad, equidad, confianza, innovación y no perder de vista los objetivos; factores que pueden determinar el éxito o fracaso de un emprendimiento.

\section{Espíritu Empresarial}

En los últimos años temas como emprendimiento, competitividad y desarrollo económico basado en ventajas competitivas han tomado suma importancia. Rodrigo Varela en su libro Innovación Empresarial, determina que al conjunto de valores que debe poseer el emprendedor se le denomina espíritu empresarial. A continuación se presentan los principales: Fuerza vital, constituye la energía, dedicación, coraje y decisión para llevar a cabo las ideas. Deseo de superación y progreso, se refiere al motor de mejoramiento continuo para obtener orientación hacia el logro. Capacidad de identificar oportunidades, entendida como la competencia de visualizar, escuchar e interpretar el entorno para detectar posibles oportunidades. Visión de futuro, constituye la capacidad de identificar tendencias. Habilidad creadora e 
innovadora, se refiere a la habilidad de proponer múltiples, variadas y apropiadas maneras de solución a los problemas y de satisfacer necesidades. Aceptación y propensión al cambio, entendidos como la flexibilidad oportuna y acorde a los retos del entorno. Iniciativa, constituye la capacidad de anticiparse a los demás (Varela, 2014).

Los valores citados anteriormente y varios más constituyen el espíritu emprendedor que es necesario para dirigir emprendimientos de éxito. El espíritu empresarial desempeña un papel básico en todo proceso de desarrollo humano y social

¿Cómo surgen los empresarios? Estos surgen de individuos con espíritu empresarial, los verdaderos iniciadores del progreso económico, los generadores de riqueza. Diversos estudios internacionales plantean que los empresarios se forman desde dos perspectivas: necesidad y oportunidad. En nuestro país la gran mayoría de emprendimientos ha surgido por necesidad.

\section{Desarrollo Económico}

El desarrollo económico como objeto de estudio en la ciencia social ha trascendido el tiempo desde los padres de la teoría económica clásica, hasta los modernos pensadores económicos, que han desarrollado desde diversos puntos de vista modelos de desarrollo económico.

El desarrollo económico tiene como objetivo generar bienestar y calidad de vida. Todos los gobiernos en su deseo profundo y ético han planteado como fin el desarrollo económico, la igualdad y la mejora en la calidad de vida. En la actualidad existe la tendencia de que los modelos económicos para el desarrollo económico se basan en el desarrollo empresarial. Por tal razón se considera que la creación de empresas, la cultura empresarial y por consiguiente, al surgimiento de nuevos empresarios como vía para generar desarrollo económico.

Hugo Kantis en su estudio denominado Desarrollo Emprendedor, indica que quienes crean riqueza son los empresarios, mientras que los estados facilitan y crean el ambiente propicio para que los emprendedores puedan crear empresas como proyectos de vida (Kantis, 2004).

Países basan sus modelos económicos, políticas e instituciones en fomentar la creación de empresas son países que obtienen crecimiento económico, desarrollo económico, mediante la generación de riqueza y el mejoramiento de la calidad de vida en sus pobladores. Mientras, aquellos que no centran sus modelos económicos de manera coordinada en la creación de empresas y mejoramiento de las ya creadas son países que no logran mantener o alcanzar la competitividad internacional y se tornan en constantes y eternos países en vías de desarrollo, forma suave de calificar contextos de subdesarrollo (Crisien C., 2006).

Frente a esto se considera indispensable que a nivel gubernamental como ente facilitador y generador de política, se encaminen los esfuerzos a todo nivel para poder desarrollar una cultura hacia el emprendimiento y la obtención de competitividad en los existentes. Además, los entes educativos, los medios de comunicación, el sistema financiero y la sociedad en general deben dirigir sus esfuerzos hacia un sistema que conduzcan a la creación de nuevas empresas y al fortalecimiento de las ya existentes. 
Según estudios realizados por Jane Jacobs, el proceso de desarrollo económico es responsabilidad de todos los integrantes de una comunidad, enfatizando en que es un proceso humano, que requiere gente preparada, lista y dispuesta para conseguirlo. Por lo cual indica que el desarrollo exige al sistema social la adquisición de actividades dinámicas como la elasticidad, creaividad e innovación, iniciativa y diversidad (Jacobs, 1983).

Amartya Sen, Premio Nobel de Economía, relaciona la concepción de desarrollo económico de un país con la teoría del desarrollo local, indicando que éste, busca que los pobladores de un territorio pueden incrementar las posibilidades de obtener oportunidades, para desarrollar procesos productivos, que les permitan generar su propio empleo y riqueza, consintiéndoles además mejorar su calidad de vida y proyectarse a futuro como generadores de valor agregado a todos los procesos que hacen parte del funcionamiento económico y social de la comunidad (Sen, 2000).

\section{Educación y Desarrollo}

Silvia Sioli de Torres Carbonel en el V Encuentro de Empresarios, desarrollado en Buenos Aires en Abril de 2002, sostuvo que si la educación es el medio por el cual se logra modificar actitudes y comportamientos, se podría decir que existe una relación absoluta entre educación y emprendimiento; por tal motivo se debería pensar en educar a los emprendedores, ya que es posible que existan muchos emprendedores que nazcan con las características necesarias para serlo, pero también existirán muchas personas que requieran desarrollar estos rasgos, por lo que se puede asegurar que todos necesitan capacitarse en esta temática (Sioli, 2002).

Por las razones antes mencionadas, se considera necesario consolidar los espacios de formación, pues las personas son receptoras de habilidades, conocimientos, actitudes y capacidades, que en gran medida se fortalecen o se debilitan dependiendo de los escenarios en los que se desenvuelven. La formación en emprendimiento constituye un factor fundamental para el desarrollo local, beneficio que la sociedad recibe al afrontar de manera más eficiente la satisfacción de las necesidades y la solución de los problemas de la comunidad oportunamente, lo que reconoce al emprendedor como figura auténtica en el progreso permanente de una localidad a nivel municipal y/o regional. (Duarte \& Ruiz, 2009)

La inversión en investigaciones relacionadas con ciencia y tecnología constituye un factor prioritario para fomentar la capacidad emprendedora, la creatividad e innovación en la población, la misma que debe ser fomentada por las instituciones de educación superior para contribuir al mercado laboral con profesionales capaces de crear y gestionar nuevos negocios, que aporten significativamente a la transformación de la matriz productiva. Por tal razón se recomienda dar el apoyo y soporte necesarios a nivel institucional para consolidar la formación en el tema de emprendimiento.

Para dar cumplimiento a lo antes mencionado se considera que las alianzas estratégicas universidad-estado constituyen una alternativa clave para la ejecución de proyectos de desarrollo empresarial y fomentar el espíritu emprendedor.

\section{Realidad ecuatoriana}


Desde 1999, se ha venido desarrollando año a año un gran proyecto de investigación a escala mundial, que permite la medición y análisis de la actividad empresarial en muchos países, proyecto denominado Global Entrepreneuroship Monitor (GEM). El informe anual del GEM Ecuador 2016 describe a los emprendedores en Ecuador, los factores que inciden en el emprendimiento, y la dinámica de creación de empresas. A continuación se citan los datos más relevantes de dicho reporte:

En el 2016, el Índice de Actividad Emprendedora Temprana (TEA) para Ecuador fue de $31.8 \%$ siendo 2.2 veces mayor al promedio de las economías de eficiencia. Esto representa una ligera disminución en la actividad emprendedora en comparación al 2015, donde la TEA de Ecuador se ubicó en 33.6\%. Pese a esto, Ecuador sigue siendo el país con la TEA más alta en la región, y el segundo entre los 66 países participantes (Global Entrepreneurship Monitor, 2016).

Un $20.8 \%$ de la población adulta afirmó haber emprendido aprovechando una oportunidad, mientras que un $8.9 \%$ lo hizo por necesidad. Entre los emprendedores por oportunidad, el $22.5 \%$ lo hizo para mejorar ingresos actuales, el 35.2\% para obtener mayor independencia y el $42.3 \%$ por ambos motivos (Global Entrepreneurship Monitor , 2016).

Las cifras presentadas anteriormente indican una tendencia a la baja, que aunque sea mínima, debe ser considerada para la aplicación de estrategias que minimicen el impacto en el crecimiento económico del país. En efecto, el crecimiento económico supone cambios culturales y sociales que hacen que la visión del emprendedor cambie, así como sus expectativas respecto al futuro.

\section{Conclusiones}

La presente investigación pretende presentar una alternativa de desarrollo y competitividad a nivel país, forjada mediante la generación de un tejido empresarial de calidad que nace de la educación empresarial.El espíritu empresarial posibilita a la comunidad alcanzar el desarrollo, pues genera una cultura enérgica, con dinamismo, orientación al trabajo, variedad, elasticidad, acción, innovación, creatividad, componentes principales de los valores culturales de una sociedad emprendedora, direccionada hacia la competitividad y tendiente a alcanzar el desarrollo económico.

Cuando se entienda que la cultura del emprendimiento es un elemento estructural para lograr competitividad y, que por ende debe abordarse desde esta óptica, se habrá superado el primer umbral para lograr un mayor nivel de crecimiento económico soportado sobre empresas competitivas. Por tanto, debido a la relevancia que tiene este factor, es de gran importancia determinar las medidas que resultan más adecuadas para estimularlo.

\section{Agradecimiento}

El presente trabajo de investigación forma parte de los resultados del proyecto de investigación "Propuestas de mejoras de la competitividad interna/empresarial de la mipyme de la Provincia de El Oro", de la Carrera Gestión Empresarial de la UMET, Sede Machala, por lo cual se agradece a la UMET por hacer posible la presente investigación.

\section{Bibliografía}


Bóveda, J. Á. (2004). El emprendedor y las pequeñas empresas. Paraguay: Revista Futuros - Fundación Emprender.

Cantillón, R. (1755). Essai Sur la Nature du Commerce en General. Londrés: UK: Macmillan. (Traduccion publicada en 1931).

Casson, M. (1982). The Entrepreneur. An Economic Theory. Oxford, Reino Unido: Martin Robertson \& Company.

Crisien C., J. (2006). Espíritu emprendedor como estrategia de competitividad y desarrollo económico. Escuela de Administración de Negocios, 103-117.

Duarte, T., \& Ruiz, M. (2009). Emprendimiento, una opción para el desarrollo. Scientia et Technica .

Ecuador), U. E. (n.d.). Retrieved 2 de diciembre de 2016 from http://providenciagye.blogspot.com/2012/04/la-unidad-educativa-de-la-providencia.html

Global Entrepreneurship Monitor. (2016). Executive Report: Ecuador. Graduate School of Management.

Jacobs, J. (1983). Entrepreneuroship Proceedings of the Community. Economic Developement, Strategies Conference, 115-135.

Kantis, H. (2004). Desarrollo emprendedor: América Latina y la experiencia internacional. Banco Interamericano de Desarrollo. FUNDES International.

Madrid, M. (2010). International Competitiveness: Measurement Indicators and Structural Aspects with Specific Reference to the Situation in Spain". International Political Economy, Nova Press, Nueva York, 91.

Mason, C., \& Brown, R. (2014). Entrepreneurial Ecosystems and Growth Oriented Entrepreneurship. París: Final Report to OECD.

Sen, A. (2000). Desarrollo y Libertad. Buenos Aires: Editorial Planeta.

Shane, S., \& Venkataraman, S. (2000). The Promise of Entrepreneurship as a Field of Research. Academy of Management Review.

Sioli, S. (2002). V Encuentro de Empresarios. Buenos Aires: Asociación Cristiana de Dirigentes de Empresas.

Soto Rodriguez, E. (2014). Entrepreneurial Ecosystems as a Pathway towards Competitiveness: The Case of Puerto Rico. Competition Forum Vol. 12, N 1, 31-40.

Taymaz, E., \& Köksal, M. (2006). El espíritu emprendedor, el tamaño de lanzamiento y la supervivencia de los pequeños empresarios. Revista vasca de economia, No. 62, 70-99.

Unidad Educativa "De La Providencia". (n.d.). Retrieved 2 de diciembre de 2016 from http://delaprovidencia.edu.ec/Main.aspx

Varela, R. (2014). Innovación Empresarial. Bogotá: Pearson. 